\title{
Cytomorphology of Chondroid Syringoma: Analysis of 20 Cases and Review of Literature
}

\author{
Meetu Agrawal, Mukul Singh, Rituraj*, Sachin Kolte, C. Ahluwalia, \\ Neha Madan and Rashmi Arora \\ Department of Pathology, Vardhman Mahavir Medical College (VMMC) and Safdarjung Hospital (SJH), New Delhi. India 110029
}

\begin{abstract}
Background: Chondroid syringomas [CS] are interesting skin adnexal tumours. They have a remarkable pleomorphic-adenoma like morphology in the skin. Though the histopathology of the tumour has been described in detail in literature, cytomorphological studies are restricted to case reports and small case series. For diagnosing a CS on cytology, the index of suspicion must be high, clinical evaluation must be thorough and knowledge of variant existing morphologies is essential.

Aim: This study was done with the aim of studying the common and variant cytological features of chondroid syringomas.

Methods:This was a retrospective study conducted over a four-year period (2014-17) in the Department of Pathology, VMMC and Safdarjung hospital. Fine needle aspirate smears and biopsy slides were studied. Morphological details were independently reviewed in detail by two pathologists (Dr MS and Dr MA).

Result and Conclusion: The conventional biphasic cell morphology of CS was seen in nearly 50\% cases. Rest of the cases showed predominance of one or more variant features in few areas and thus CS was only one of the differential diagnosis in these cases. Nevertheless, FNA proved to be fast, inexpensive and a reliable method for diagnosis of chondroid syringoma. The study will be relevant and helpful in the day to day cytological reporting of CS.
\end{abstract}

Keywords: Chondroid Syringoma, Skin Adnexal Tumors, Cytomorphological Variation, Chondromyxoid.

\section{Introduction}

Chondroid syringomas $[\mathrm{CSs}]$ are benign skin adnexal tumors. ${ }^{1}$ There is enough morphological evidence to believe that they are derived from both ductal and acinar components. $^{2}$ The epithelial components resemble sweat glands. ${ }^{1}$ However, it is unclear whether they actually originate from the eccrine or apocrine glands. ${ }^{3,4}$ The CSs are histologically analogous to pleomorphic adenomas of the salivary glands. Abundant data exists on the latter, however there is scarce literature on detailed cytological features of CS. Cytological descriptions of CS have been restricted to case reports and short case series. The diagnosis may be missed on cytology, considering the various morphological variations which can exist. This article presents a detailed report of 20 cases, with a careful analysis of common and rare cyto-morphological features.

\section{Materials and Method}

A retrospective study was conducted over a four-year period (2014-17). Cases diagnosed as chondroid syringomas were taken out from the pathology database of the Department of Pathology, VMMC and Safdarjung hospital. Corresponding fine needle aspirate smears and biopsy slides were studied. Demographic and clinical details of patients were collected from the cytology requisition forms and patient files. Cases which were wrongly diagnosed as CS on FNAC or those which did not have any histopathological correlation were excluded from this study.

In most cases FNAC had been performed by a 23-gauge needle, mounted on a Franzen handle. Both Papanicolaou and Giemsa stained smears were studied wherever available. Each case had an average of four slides (range 3-8). Morphological details were independently reviewed in detail by two pathologists (Dr MS and Dr MA). Cytomorphological details evaluated for each case includedcellularity, matrix, cell morphology and associated metaplasia, calcification and special features, if any. All cases had histopathological correlation.

\section{Results}

During the study period, 209 cases were diagnosed as skin adnexal tumors on cytology. Of these, only 104 cases had follow-up excision specimen. Most cases (51/104; 49\%) have been reported as skin adnexal tumors (47-benign and 04 malignant). After a complete histo-cytological correlation, the number of CS included in the study was 20. Of these 14 cases were diagnosed as CS at the time of primary diagnosis. In four out of the remaining six biopsy confirmed cases, the first diagnosis was benign skin adnexal tumor and CS had been suggested as a differential 
diagnosis. Two of the cases were earlier diagnosed as epidermal inclusion cysts. One of these cases showed abundant squames and the other showed presence of nucleate and anucleate squames along with prominence of calcification.

The mean age of presentation was 34.2 years (range: 19-73 years). There was male preponderance with a male: fem ale ratio of 2.2:1. The commonest site was upper extremity [07/20 cases- $35 \%$; followed by cheek in 5/20 (25\%) and dorsum of nose in $03 / 20(15 \%)$ cases. Other uncommon sites included eyelids ( 2 cases), leg ( 1 case) and axilla ( 2 cases). [Fig 1] All of these lesions (including cheek) presented as raised skin nodules which were microscopically centered in the dermis. For the cheek lesions, a diagnosis of CS was preferred due to the clinical appearance of the lesion. Overall, all 20 cases had interpretable aspirates. Of these, fifteen cases had smears with moderate to high cellularity and five were paucicellular. Ten out of twenty $(50 \%)$ cases had identifiable biphasic pattern (epithelial and myoepithelial cells) lying against a chondromyxoid matrix [Fig 2(a)]. The cells had uniform bland nuclei, inconspicuous nucleoli and moderate cytoplasm. These features were present variably in different proportions in slides, but were demonstrable. No mitosis, cytologic atypia or necrosis was noted.

Of the remaining ten cases, cells were either spindle shaped or cuboidal. Apart from the classical morphology, other strikingly noticeable morphological feature was the presence of plasmacytoid cells in 10/20 (50\%) cases. [Fig 2(b)] Other unusual features were the predominant presence of multivacuolated (sebaceous like) [Fig 3(a)], glandular pattern [Fig 3(b)], and ghost cells [Fig 4(a)], in $4 / 20,3 / 20$ and $1 / 20$ cases respectively. However, chondromyxoid matrix was present at least focally in eight of these ten cases.

Extensive squamous metaplasia, [Fig 4(b)] one showing foci of calcification [Fig 5(a)] was seen in histopathology in two cases. There was no evidence of any chondromyxoid matrix on cytology in either of these cases. Both these cases had been earlier diagnosed as epidermal inclusion cysts on cytology.

For most cases, histopathological analysis showed variable sized tubules lined by double layer of cells, inner cuboidal and outer flattened. [Fig 5(b)] In cases with extensive variant morphology on cytology, focal changes such as presence of multivacuolated cells was seen but also present was classical morphology with abundant chondromyxoid stroma which was present in $18 / 20$ cases. In the two cases diagnosed as epidermal inclusion cysts, squamous metaplasia and calcifications were seen, with many areas showing classic morphology of CS.

Thus, overall cyto-histological concordance was seen in 18/20 (90\%) cases.

All the above findings are depicted in Figure 2 and summary of these results is seen in Table 1.

\section{Discussion}

Fine needle aspiration cytology is often the preliminary diagnostic modality in most skin adnexal tumors, including CS. Though the morphological features on histopathology are well understood and described, cytology descriptions have been mainly restricted to individual case reports and

Table 1: Various cyto-morphological features observed in this study.

\begin{tabular}{|c|c|c|c|}
\hline \multicolumn{2}{|l|}{ FEATURES } & \multicolumn{2}{|c|}{ STATISTICS } \\
\hline \multirow{2}{*}{ Cellularity } & Moderate to high cellularity & $15 / 20$ & $75 \%$ \\
\hline & Paucicellular & $5 / 20$ & $25 \%$ \\
\hline \multirow{8}{*}{ Cytomorphology } & $\begin{array}{l}\text { Predominant Biphasic pattern (epithelial and } \\
\text { myoepithelial cells against chondromyxoid background) }\end{array}$ & $10 / 20$ & $50 \%$ \\
\hline & Plasmacytoid cells & $10 / 20$ & $50 \%$ \\
\hline & Spindle cell clusters & $5 / 20$ & $25 \%$ \\
\hline & Cuboidal cell clusters & $5 / 20$ & $25 \%$ \\
\hline & Multivacuolated (Sebaceous like cells) & $4 / 20$ & $20 \%$ \\
\hline & Ghost cells & $3 / 20$ & $15 \%$ \\
\hline & Foci of calcification & $2 / 20$ & $10 \%$ \\
\hline & Squamous metaplasia & $2 / 20$ & $10 \%$ \\
\hline
\end{tabular}




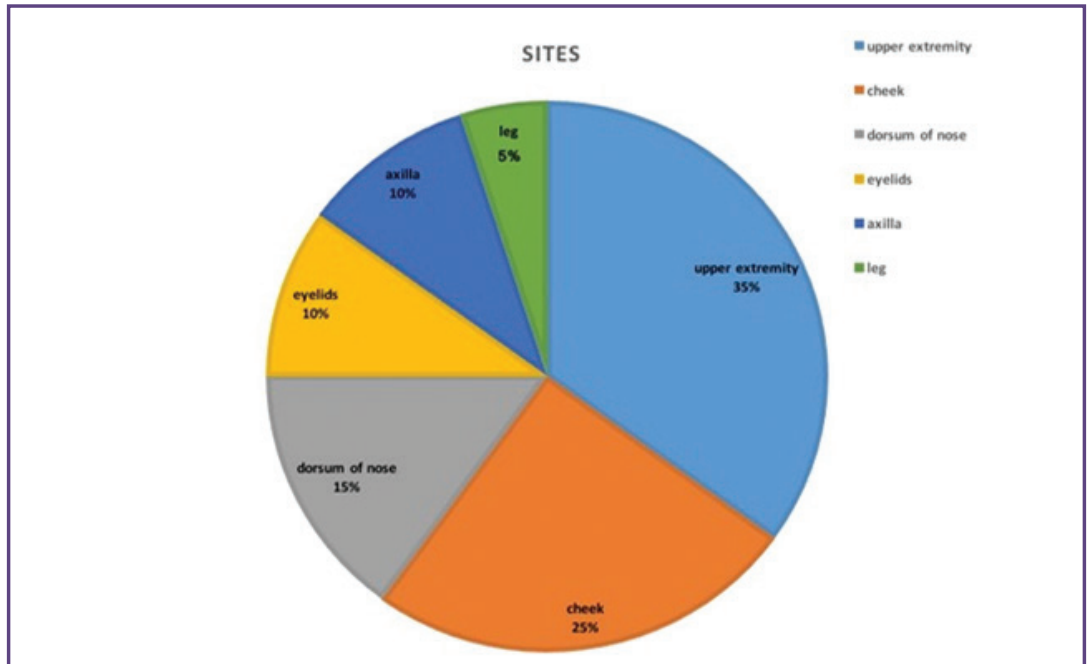

Fig. 1: Distribution of cases as per site.

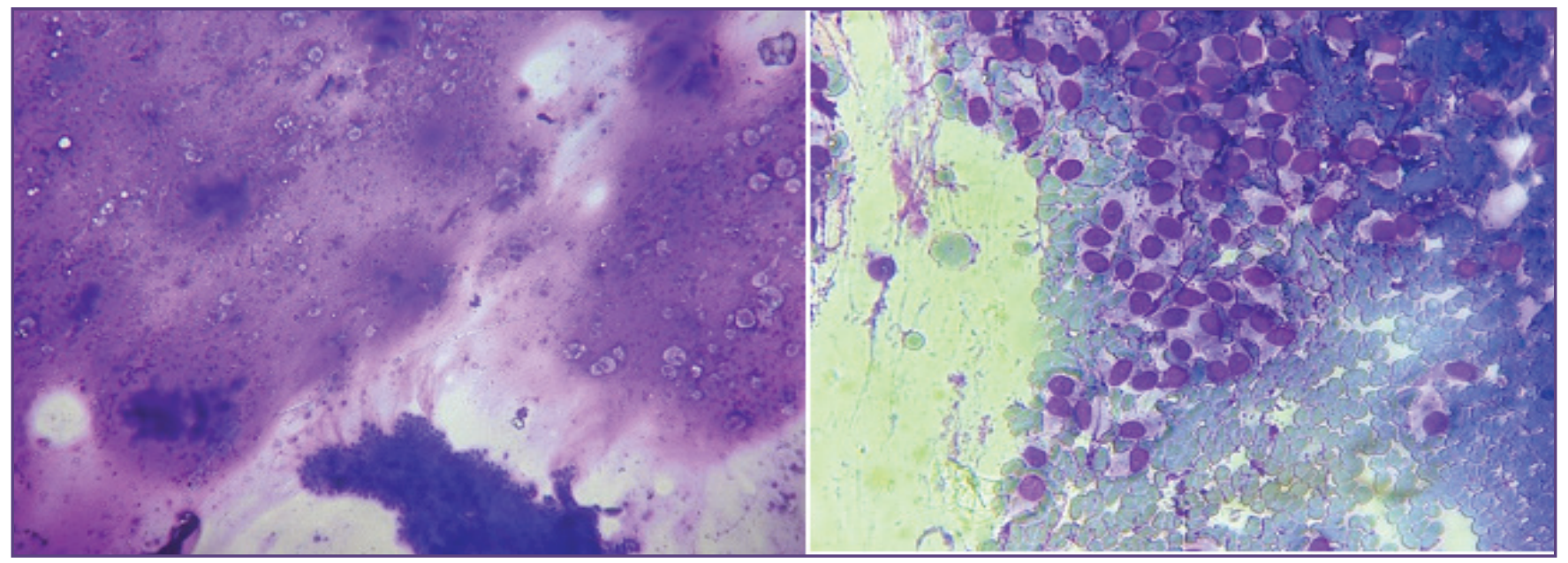

Fig. 2: (a) Biphasic pattern: Epithelial and myoepithelial cell clusters against a fibrillary chondromyxoid background (10X, Geimsa), (b) plasmacytoid cells (40x, Geimsa).

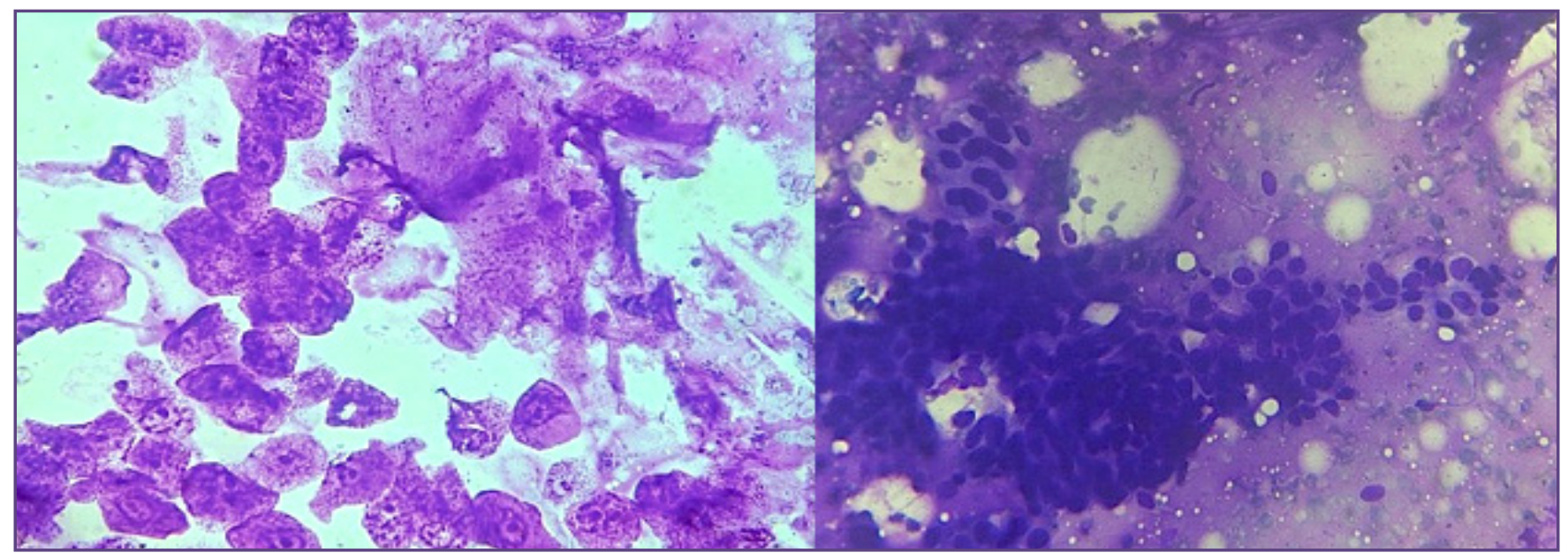

Fig. 3: (a) sebaceous like cells- multivacuolated (40x, Geimsa), (b) glandular or acinar pattern of cells (40x, Geimsa). 


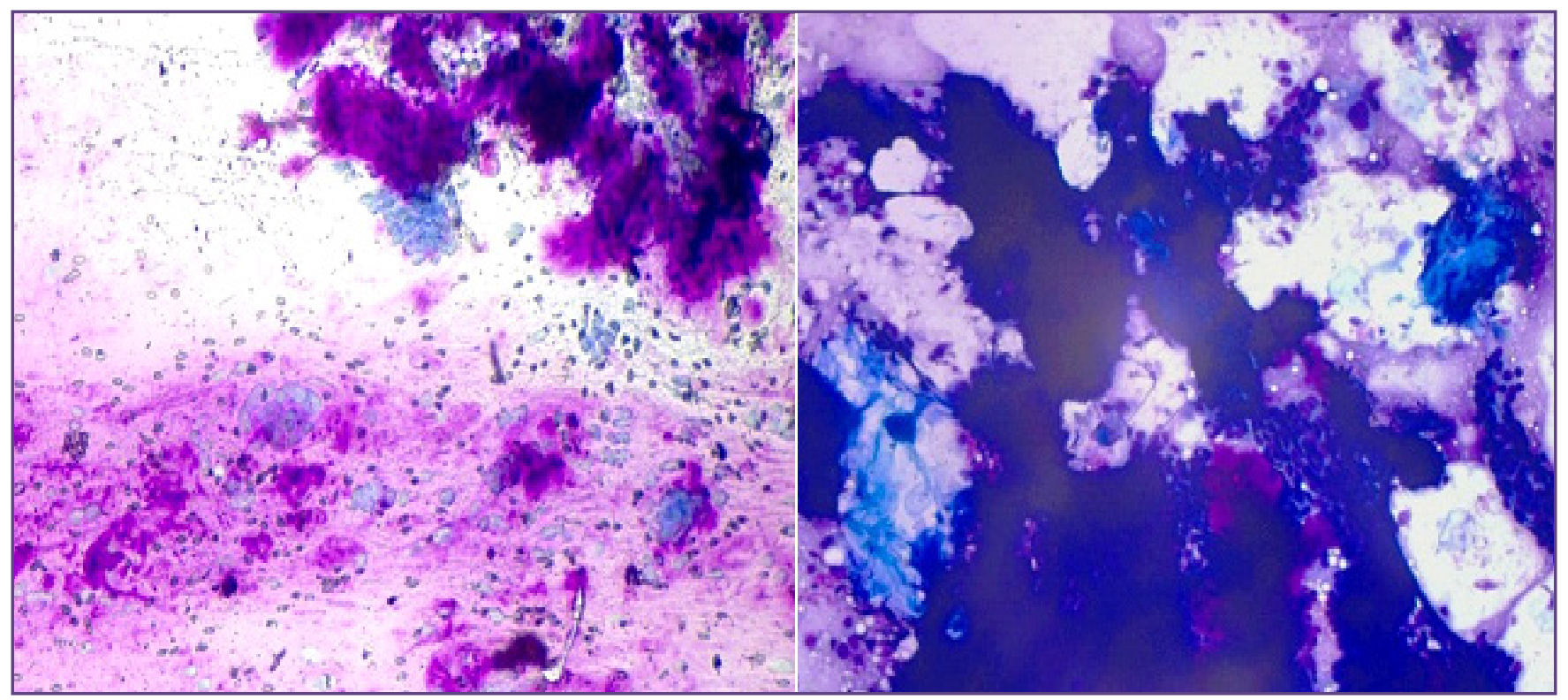

Fig. 4: (a) ghost cells (20x, Geimsa), (b) areas with squamous metaplasia (40x, Geimsa).

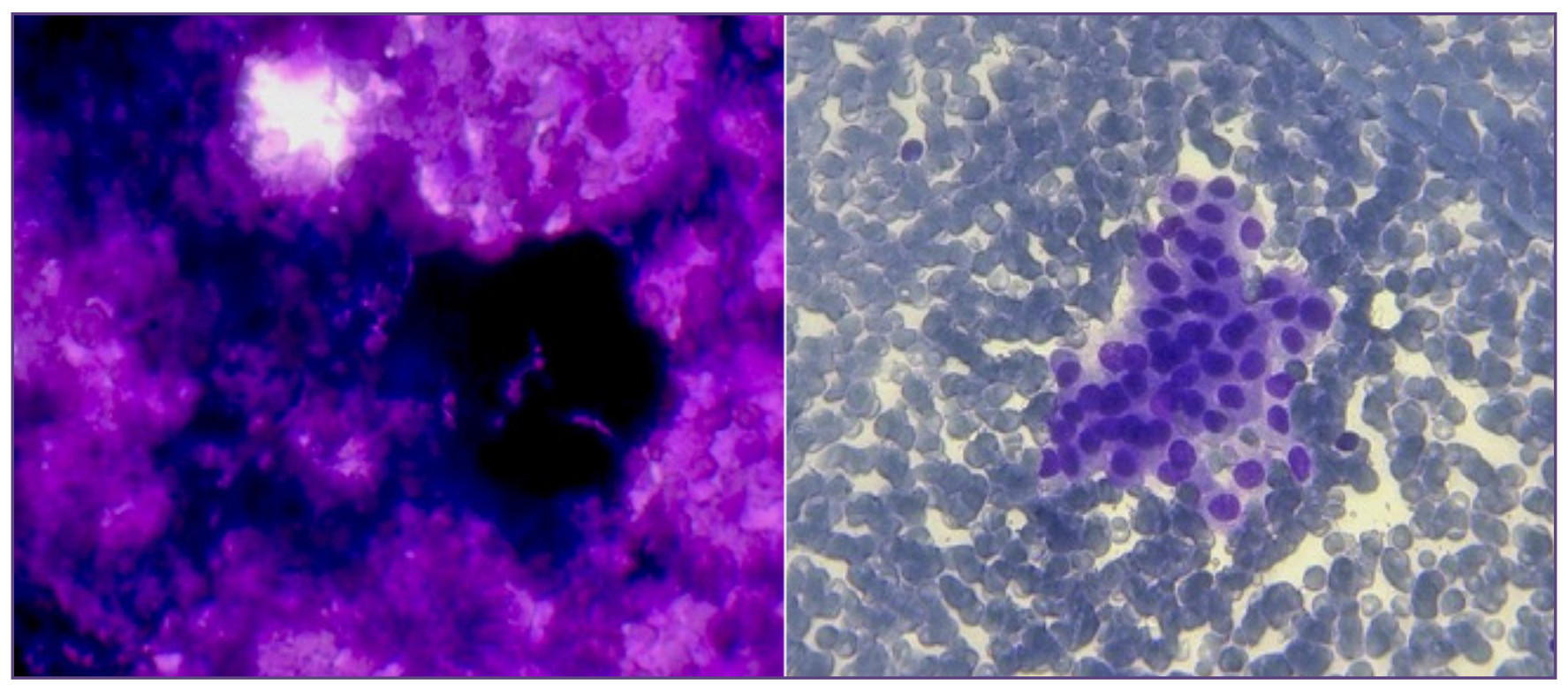

Fig 5: (a) areas of calcification (40x, Geimsa). (b) cuboidal cells (40x, Geimsa).

small case series. In the background of paucity of data on this particular subset of benign skin adnexal tumors, the present study provides a detailed and till-date largest cytological analysis of CS.

The presentation of CS is not specific. Most of the cases included in this study presented as painless superficial skin nodules with overlying unremarkable skin. There was no functional impairment except for one case located on the eyelid which slightly lead to restricted mobility of the lid. The findings of this study are in accordance with those reported in literature.
CS normally present in regions such as scalp, nose and axilla; sites such as upper lip and breast have also been reported.

Upper extremity is an uncommon site; in this study however, lesions were commonly found in the arms (lower arm and hand). An explanation for this may be attributed to sheer coincidence or the fact that the prior studies were either individual cases or small case series. Texture and consistency of the aspirate is usually mostly thick mucoid which relates to the abundant chondromyxoid matrix. However, superimposed cystic 
change can give rise to a more fluid consistency with scant chondromyxoid matrix. ${ }^{8}$

$\mathrm{CS}$ is the skin adnexal counterpart of pleomorphic adenoma in the salivary gland. Cyto-morphological features commonly seen in CS are presence of epithelial and myoepithelial cells in a chondromyxoid matrix. Presence of other features, as was seen in this study is highly variable. One or more of these variable features, either independently or their congregation predominate in individual cases. These are the cases where diagnosis may be missed on cytology. Here important differential diagnosis such as sebaceous cyst, dermoid cyst, basal cell carcinoma with mucinous stroma, fibro myxoid sarcoma or pilomatrixoma have to be considered or ruled out., Nodular fasciitis and benign neve sheath tumors must be considered in cases where there is predominant population of spindle shaped cells. Nodular fasciitis is characterized primarily by the presence of spindle cells with appreciable anisonucleosis and multinucleation. ${ }^{6}$ Buckling of slender nuclei can be seen in at least some cells in an aspirate from benign nerve sheath tumours. Malignancy usually does not pose a problem as cytological features are obvious.

Classic biphasic pattern with a chondromyxoid matrix was seen in only $50 \%$ cases in this study. For rest of the $8 / 20(40 \%)$ cases, variant histomorphology was seen. Nevertheless, careful and diligent search for the presence of chondromyxoid matrix in FNA smears provided strong clues to the diagnosis. A careful search for presence of typical biphasic pattern and/or chondromyxoid matrix does help to clinch the diagnosis as in many of our cases.

A substantial fraction of our cases $(30 \%)$ was also seen in the cheek. Four out these six cases had classical morphology, one case showed prominence of plasmacytoid cells and another was paucicellular with prominence of chondromyxoid matrix. These cases were still categorised as chondroid syringoma and not pleomorphic adenomas. Though microscopically indistinguishable, presence of these lesions as raised skin nodules, a dermo-centric location rather than their presence in the substance of the cheek favored CS over pleomorphic adenoma. There was no salivary gland tissue seen either as part or surrounding the tumor.

The presence of plasmacytoid cells as a predominant feature is seen in many cases of CS. Presence of plasmacytoid cells as a prominent feature is also seen in myoepitheliomas of the soft tissue. However, myoepitheliomas are very rare tumours located in deep soft tissue and thus can be clinically differentiated from CS by the plane of location. ${ }^{7}$

Extremities have been reported to be common sites for malignant transformation in CS with the frequency of malignant change being as high as $82 \%{ }^{3}$ The commonest site in our study was extremities; however, till date none of the patients in our study had any evidence of recurrence or malignant change.

Chondroid syringomas of both apocrine or eccrine types have been beautifully described by Headington JT. ${ }^{9}$ Whereas on one hand the apocrine lesions have a double layered epithelium, the eccrine ones are characterized by a single layer of epithelial cells in a background of abundant chondro-myxoid matrix. Both the types were seen in our study.

The treatment of choice in CSs is surgical excision with wide margin. Incomplete removal can lead to recurrence; infrequently malignant transformation may be seen.

FNAC offers many advantages in terms of being fast, inexpensive and often diagnostic. To the best of our knowledge, this is the largest study on cytology of chondroid syringomas.

\section{Conclusion}

The morphological features of CSs are very well defined in histopathology. Cytology however presents some diagnostic challenges. This could be due to sampling errors or when one component predominates over the others.

This paper presents a collection of few cases of cytology of CSs which should be kept in mind while reporting these cases.

\section{Acknowledgements}

Special thanks to Dr. Rashmi Arora, Head of department pathology, VMMC and SJH, New Delhi for her support and guidance.

This paper is not funded and there is no conflict of interest.

\section{References}

1. Lamba S, Nanda A, Kumar U. Chondroid Syringoma: Fine-needle Aspiration Cytology of a Rare Entity at an Unusual Site. Journal of Clinical and Diagnostic Research. 2017;11:ED06-ED07.

2. Kundu R, Punia RS, Handa U, et al. Chondroid syringoma: Cytomorphology of four cases and review of literature. Archives of Cytology and Histopathology Research. 2016;1:63-7.

3. Harrist TJ, Aretz TH, Mihm MC Jr, et al. Malignant chondroid syringoma. Arch Dermatol. 1981;117:719-24.

4. Hirsch P, Helwig EB. Chondroid syringoma: Mixed tumour of skin, salivary gland type. Arch Dermatol. 1961;84:83547.

5. Yavuzer R, Basterzi Y, Sari A, et al. Chondroid syringoma: a diagnosis more frequent than expected. Dermatol Surg. 2003;29:179-81 
6. Silvanto AM, Melly L, Hannan SA, et al. FNAC of nodular fasciitis mimicking a pleomorphic adenoma: another diagnostic pitfall. Cytopathology. 2010;21:276-7.

7. Moon A, Chin S, Kim HK, et al. Myoepithelioma of soft tissue in the knee: A case report of an unusual location. Indian J Pathol Microbiol. 2016;59:254-6.
8. Khan K. Chondroid syringoma: a case with unusual cytological findings. Indian J Dermatol. 2013;58:157.

9. Headington JT. Mixed tumours of skin: eccrine and apocrine types. Arch Dermatol. 1961;84:989-96.

\footnotetext{
*Corresponding author:

Dr. Rituraj, Room no 416, college building, 4th floor VMMC and SJH, New Delhi. India, Pin code: 110029

Phone: +91 7042636607

Email: 04rraj04@gmail.com
}

Financial or other Competing Interests: None. 